\title{
VUV PHOTOCHEMISTRY OF PAHS TRAPPED IN INTERSTELLAR WATER ICE
}

\author{
J. Bouwman ${ }^{1}$, H.M. Cuppen ${ }^{1}$, L.J. Allamandola ${ }^{2}$ and H. Linnartz ${ }^{1}$
}

\begin{abstract}
The mid-infrared emission of Polycyclic Aromatic Hydrocarbons is found in many phases of the interstellar medium. Towards cold dense clouds, however, the emission is heavily quenched. In these regions molecules are found to efficiently freeze-out on interstellar grains forming thin layers of ices. PAHs are highly non-volatile molecules and are also expected to freeze-out. PAHs trapped in interstellar ices are likely to participate in the overall chemistry, leading to the formation of cations and complex molecules in the solid-state. The work presented here aims to experimentally study the chemical reactions that PAHs undergo upon vacuum ultraviolet irradiation when trapped in interstellar $\mathrm{H}_{2} \mathrm{O}$ ice.
\end{abstract}

\section{Introduction}

PAHs are abundantly present in many phases of the ISM as evidenced by their midIR emission bands. Towards dense clouds, however, this emission process is heavily quenched. A possible explanation is that PAHs freeze-out on cold interstellar grains, much like small molecules such as $\mathrm{H}_{2} \mathrm{O} \mathrm{CO}, \mathrm{CO}_{2}$, and $\mathrm{CH}_{3} \mathrm{OH}$, which have been identified in interstellar ices. Once trapped in ices, PAHs are expected to absorb mid-IR photons, rather than emitting them.

Most PAH species have been studied spectroscopically in matrix isolation experiments. Pioneering work has been performed in the past to understand the chemical behavior of PAHs trapped in interstellar ice analogues; the formation of new PAH based products was studied by Bernstein et al. (1999) and the efficient ionization of PAHs in $\mathrm{H}_{2} \mathrm{O}$ ice was first noted by Gudipati \& Allamandola (2003). Here, we build on these first findings by employing near-UV/VIS absorption spectroscopy to present ionization and photoproduct formation rate constants for a

${ }^{1}$ Raymond and Beverly Sackler Laboratory for Astrophysics, Leiden Observatory, Leiden University, PO Box 9513, 2300 RA Leiden, The Netherlands

2 NASA-Ames Research Center, Space Science Division, Mail Stop 245-6, Moffett Field, CA 94035, USA 


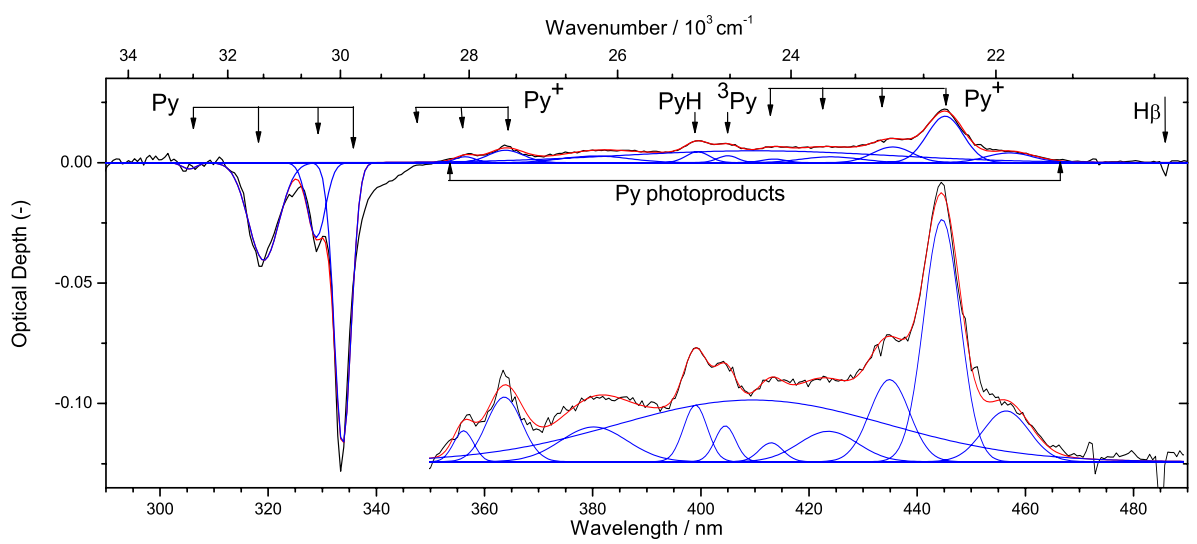

Fig. 1. A typical near-UV/VIS absorption spectrum of the PAH pyrene (Py) trapped in $\mathrm{H}_{2} \mathrm{O}$ ice photolyzed for $900 \mathrm{~s}$ at a temperature of $125 \mathrm{~K}$. Assignments of the destroyed (negative signal) neutral Py bands and newly formed (positive signal) Py photoproduct bands, the Py cation $\left(\mathrm{Py}^{+}\right)$and 1-hydro-1-pyrenyl radical $(\mathrm{PyH})$ are indicated by arrows. The inset shows a blow-up of the 350 to $490 \mathrm{~nm}$ range. Figure is taken from Bouwman et al. (2010).

number of PAHs trapped in $\mathrm{H}_{2} \mathrm{O}$ ice. Additionally, the photoproducts are also identified in-situ by mid-IR spectroscopy. The results are used to derive an upper limit for the possible contribution of PAHs to the 5-8 $\mu \mathrm{m}$ absorption complex towards protostellar objects.

\section{Experimental}

The PAH: $\mathrm{H}_{2} \mathrm{O}$ ice photolysis experiments are performed on two different setups, which have been described in much detail in Bouwman et al. (2009) and Hudgins et al. (1994) and will only be summarized here briefly. Both setups are based on the same principle and only differ in sample preparation and spectroscopic technique. The systems consist of a high-vacuum chamber pumped down to a pressure of $\sim 10^{-7}$ mbar. A cold finger containing a sample window is suspended in the vacuum chamber and is cooled down to a temperature of $15 \mathrm{~K}$ by means of a closed cycle He refrigerator. The sample temperature can be controlled by means of resistive heating.

$\mathrm{PAH}: \mathrm{H}_{2} \mathrm{O}$ ice samples are grown on the cold sample window by vapor codepositing milli-Q grade $\mathrm{H}_{2} \mathrm{O}$ and the $\mathrm{PAH}$ under investigation. The PAHs anthracene (Ant, $\mathrm{C}_{14} \mathrm{H}_{10}$, Aldrich, 99\%), pyrene (Py, $\mathrm{C}_{16} \mathrm{H}_{10}$, Aldrich, 99\%), or benzo[ghi]perylene $\left(\mathrm{B}_{\text {ghi }} \mathrm{P}, \mathrm{C}_{22} \mathrm{H}_{12}\right.$, Aldrich, $98 \%$ ) for the mid-IR and additionally coronene (Cor, $\mathrm{C}_{24} \mathrm{H}_{12}$, Aldrich, 99\%) for the near-UV/VIS are used as commercially available and brought in the gas phase by heating a sample container to a temperature which is just high enough to create the desired $\mathrm{PAH}: \mathrm{H}_{2} \mathrm{O}$ sample 


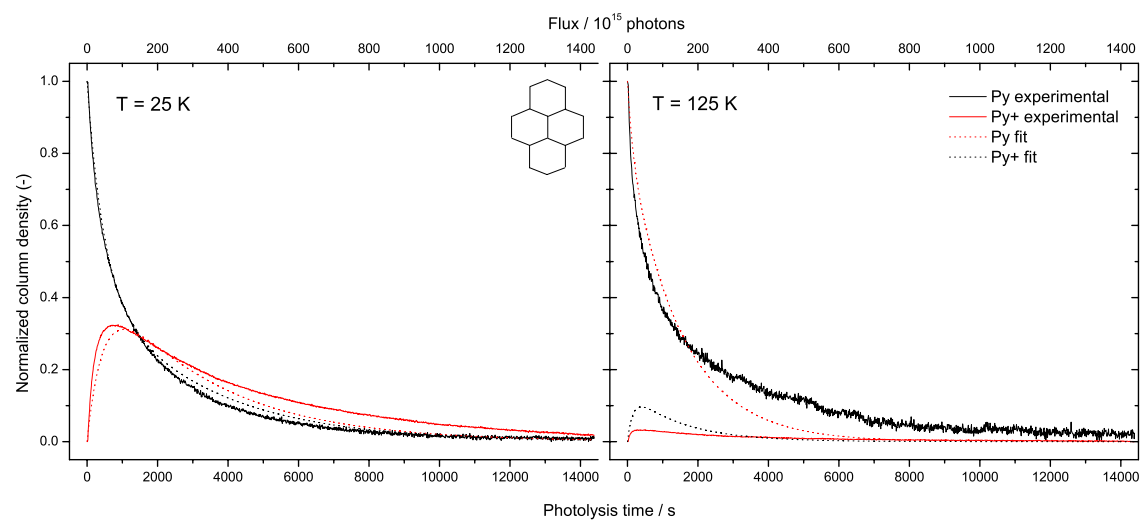

Fig. 2. Time evolution of the relative column density of neutral pyrene (Py) and the pyrene cation $\left(\mathrm{Py}^{+}\right)$in the ice as a function of photolysis time (VUV fluence). Based on data from Bouwman et al. (2010).

ratio. Mixing ratios vary from 1:10 to 1:200 for the mid-IR experiments to 1:500 to $1: 10000$ for the optical studies.

The ices for both experimental methods are subsequently irradiated with a vacuum ultraviolet (VUV) $\mathrm{H}_{2}$ microwave discharge light source to simulate the interstellar radiation field. For the mid-IR spectra, ices are irradiated for 5, 10, 15, 30, 50, 120, and 180 minutes and after each VUV dose one spectrum ranging from 4000 to $400 \mathrm{~cm}^{-1}$ is taken at $0.5 \mathrm{~cm}^{-1}$ resolution. In the near-UV/VIS experiment, spectra are recorded over the spectral range from 280 to $800 \mathrm{~nm}$ with a resolution of $0.9 \mathrm{~nm}$.

\section{Near-UV/VIS absorption spectroscopy}

Near-UV/VIS absorption spectra are obtained for a set of VUV processed PAH: $\mathrm{H}_{2} \mathrm{O}$ ices (Bouwman et al. 2011a). A typical example of a spectrum is shown in Figure 1. This spectrum is obtained by taking the ratio of a spectrum after photolysis time $t$ and a spectrum taken of the freshly deposited ice $\ln \left(I_{t} / I_{0}\right)$. The resulting spectrum exhibits positive absorptions arising from the newly formed species and negative absorptions arising from the consumed neutral parent PAH species. Clearly, new bands appear in the spectrum which can be mainly assigned to electronic transitions of the cationic PAH species.

Acquiring a spectrum, such as that displayed in Figure 1, takes $10 \mathrm{~s}$ and as many as 1440 spectra are obtained over the course of a single 4 hour photolysis experiment. Currently, optical spectra are available for VUV irradiated Anthracene: $\mathrm{H}_{2} \mathrm{O}$, Pyrene: $\mathrm{H}_{2} \mathrm{O}$, BenzoghiPerylene: $\mathrm{H}_{2} \mathrm{O}$, and Coronene: $\mathrm{H}_{2} \mathrm{O}$ (Bouwman et al. 2009, 2010, 2011a) ice mixtures. These data allow us to derive the time evolution of the column density of $\mathrm{PAH}$ and $\mathrm{PAH}^{+}$species in the ice as indicated in Figure 2 for a $\mathrm{Py}$ containing $\mathrm{H}_{2} \mathrm{O}$ ice. Both neutral parent $\mathrm{PAH}$ and $\mathrm{PAH}^{+}$cation species 


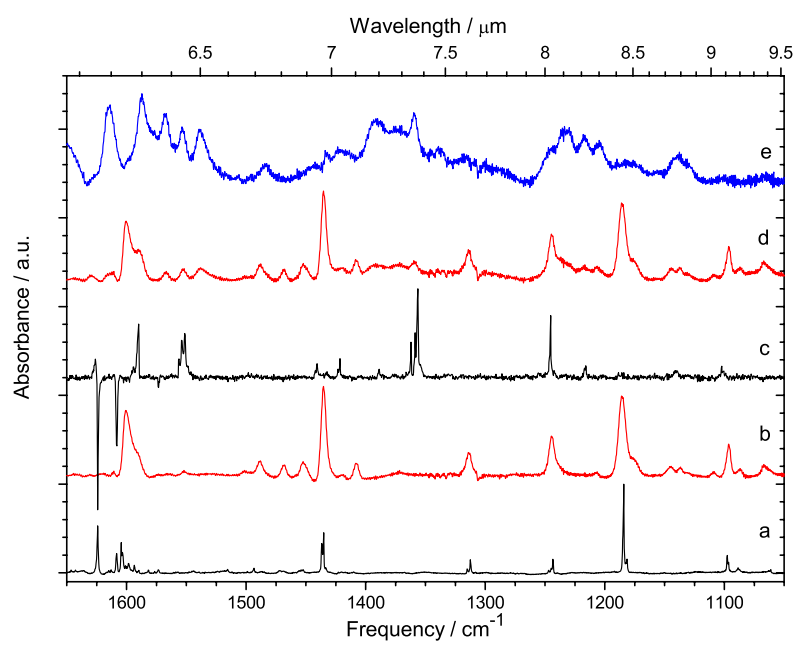

Fig. 3. Comparison between the mid-IR spectra of pyrene (Py) and pyrene cation $\left(\mathrm{Py}^{+}\right)$ in an Argon matrix ( $a$ and $c$ ) and the same spectra for Py and Py photoproducts trapped in $\mathrm{H}_{2} \mathrm{O}$ ice $(b$ and $d)$. The top spectrum $(e)$ indicates the subtraction spectrum $(d-n \times b)$ which exhibits photoproducts that appear upon photolysis of a $\mathrm{Py}: \mathrm{H}_{2} \mathrm{O}$ ice only. Figure is based on data from Bouwman et al. (2011b).

are consumed at the end of an experiment. Together with the loss of the PAH and $\mathrm{PAH}^{+}$species a broad absorption superposed on the baseline arises, which most likely makes up the absorption by the newly formed photoproducts.

A time-dependent chemical analysis is performed for all PAH species mentioned above. A chemical model is fitted to the experimental data and the rate constants for processing PAHs in an interstellar ice analogue are derived. It is found that the rate constant for ionization of PAHs in $\mathrm{H}_{2} \mathrm{O}$ ice is quite similar for all PAHs under investigation here. The derived average PAH ionization rate constant $\left(\bar{k}_{\text {ion }} \approx\right.$ $9 \times 10^{-18} \mathrm{~cm}^{2}$ photon $^{-1}$ ) can be used to quantify the importance of PAH ionization in the interstellar case by implementing this rate constant in astrochemical models.

\section{Mid-IR absorption spectroscopy}

Mid-IR spectroscopy is performed on a set of VUV photolyzed PAH containing $\mathrm{H}_{2} \mathrm{O}$ ices (Bouwman et al. 2011b). Spectra are taken on the freshly deposited ice (spectrum $X$ ) and after a certain dose of VUV light (spectrum $Y$ ). A typical spectrum of a neutral $\mathrm{PAH}$ in $\mathrm{H}_{2} \mathrm{O}$ ice, photolyzed $\mathrm{PAH}$ in $\mathrm{H}_{2} \mathrm{O}$ ice and a photolyzed $\mathrm{PAH}$ in $\mathrm{H}_{2} \mathrm{O}$ ice minus the contribution of the remaining neutral PAH species, $n$ (photoproducts solely $=Y-n X$ ) is displayed in Figure 3 for $\mathrm{Py}: \mathrm{H}_{2} \mathrm{O}$ ice. The factor $(1-n)$ directly reflects the amount of consumed neutral species.

The spectra of the photoproducts that appear upon photolysis of the PAH in $\mathrm{H}_{2} \mathrm{O}$ ice are compared to known spectra of $\mathrm{PAH}^{+}$species measured in an $\mathrm{Ar}$ 


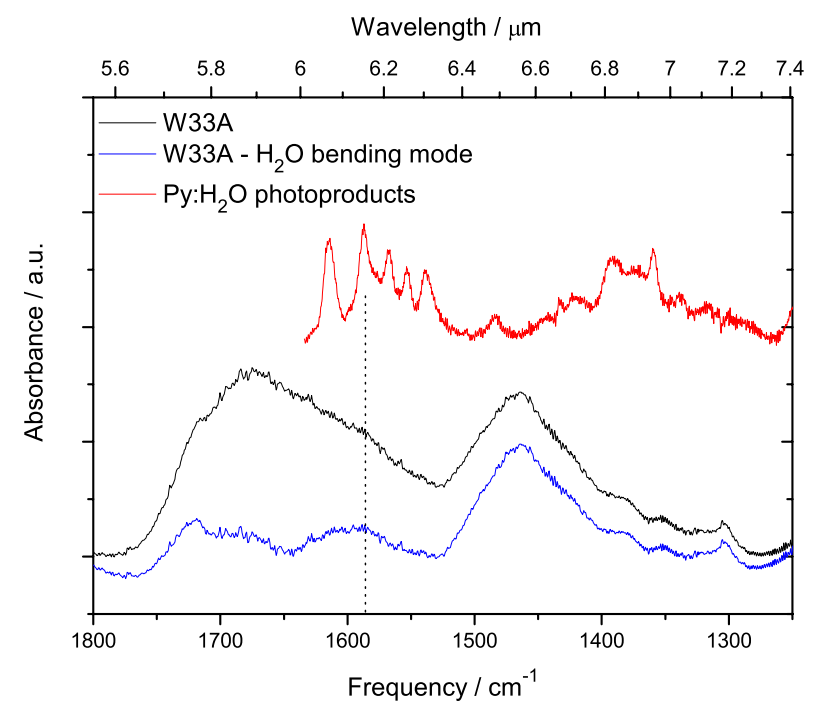

Fig. 4. Comparison between the residuals in the $5-8 \mu \mathrm{m}$ spectral region towards W33A (Keane et al. 2001) and the photoproducts formed by long duration photolysis of a pyrene containing $\mathrm{H}_{2} \mathrm{O}$ ice. The dotted line guides the eye to the $6.2 \mu \mathrm{m}$ interstellar unidentified residual absorption and the cluster of the main pyrene: $\mathrm{H}_{2} \mathrm{O}$ photoproduct bands.

matrix (Hudgins et al. 1995a, 1995b). In this way, some of the absorption bands can be directly assigned to mid-IR absorptions of the PAH cation. Other bands, however, must be caused by different band carriers.

A comparison of the new absorption bands originating from unknown species with typical mid-IR band positions indicates that the species responsible for the absorptions are likely the parent PAH with side-groups attached. In agreement with an off-site analysis of the photoproduct species resulting from the photolysis of $\mathrm{PAH}: \mathrm{H}_{2} \mathrm{O}$ ices (Ashbourn et al. 2007; Bernstein et al. 1999), we attribute these absorptions to hydrogenated, hydroxylated and oxydized PAH species.

\section{Astrophysical implications}

Many interstellar ices have been unambiguously identified by their mid-IR absorption bands observed towards protostellar objects or background stars behind dense clouds. Most of these species are rather simple molecules, such as $\mathrm{H}_{2} \mathrm{O}, \mathrm{CO}, \mathrm{CO}_{2}$, $\mathrm{NH}_{3}$, and $\mathrm{CH}_{3} \mathrm{OH}$ (Gibb et al. 2000). PAHs frozen out on interstellar grains have not yet been unambiguously identified. Here we derive from the presented spectra an upper limit for the contribution that PAH species may have to the complex absorption in the 5-8 $\mu \mathrm{m}$ mid-IR spectral range towards both high- and low-mass protostars. 
The $5-8 \mu \mathrm{m}$ spectral region is dominated by the strong $\mathrm{H}_{2} \mathrm{O}$ bending mode. The residuals that arise after subtraction of the contribution of the $\mathrm{H}_{2} \mathrm{O}$ bending mode show that more species are responsible for the overall absorption. As can be seen in Figure 4, there is a clear overlap between the residuals of W33A (taken from Keane et al. 2001) and the photoproduct bands that appear upon prolonged irradiation of a $\mathrm{PAH}: \mathrm{H}_{2} \mathrm{O}$ ice mixture. A band strength can be estimated for the $\mathrm{PAH}: \mathrm{H}_{2} \mathrm{O}$ photoproduct, because the number of molecules responsible for this band is known. This band strength is used to determine the number of molecules being responsible for the $6.2 \mu \mathrm{m}$ band (Fig. 4) if these absorption bands are indeed caused by $\mathrm{PAH}: \mathrm{H}_{2} \mathrm{O}$ photoproducts. In Bouwman et al. (2011b), an upper limit of $2-3 \%$ with respect to $\mathrm{H}_{2} \mathrm{O}$ is derived for the contribution of PAHs to the $5-8 \mu \mathrm{m}$ absorption complex.

This work is financially supported by NASA's Laboratory Astrophysics and Astrobiology Programs, "Stichting voor Fundamenteel Onderzoek der Materie" (FOM), and "the Netherlands Research School for Astronomy" (NOVA). J. Bouwman gratefully acknowledges the "Search for Extraterrestrial Intelligence" (SETI) institute for financial support.

\section{References}

Ashbourn, S.F.M., Elsila, J.E., Dworkin, J.P., et al., 2007, Meteorit. Planet. Sci., 42, 2035

Bernstein, M.P., Sandford, S.A., Allamandola, L.J., et al., 1999, Science, 283, 1135

Bouwman, J., Cuppen, H.M., Allamandola, L.J., \& Linnartz, H., 2011a, A\&A, accepted

Bouwman, J., Cuppen, H.M., Bakker, A., Allamandola, L.J., \& Linnartz, H., 2010, A\&A, $511, \mathrm{~A} 33+$

Bouwman, J., Mattioda, A.L., Allamandola, L.J., \& Linnartz, H., 2011b, A\&A, 525, A93

Bouwman, J., Paardekooper, D.M., Cuppen, H.M., Linnartz, H., \& Allamandola, L.J., 2009, ApJ, 700, 56

Gibb, E.L., Whittet, D.C.B., Schutte, W.A., et al., 2000, ApJ, 536, 347

Gudipati, M.S., \& Allamandola, L.J., 2003, ApJ, 596, L195

Hudgins, D.M., \& Allamandola, L.J., 1995a, J. Phys. Chem., 99, 3033

Hudgins, D.M., \& Allamandola, L.J., 1995b, J. Phys. Chem., 99, 8978

Hudgins, D.M., Sandford, S.A., \& Allamandola, L.J., 1994, J. Phys. Chem., 98, 4243

Keane, J.V., Tielens, A.G.G.M., Boogert, A.C.A., et al., 2001, A\&A, 376, 254 